\title{
Investigation on Locking and Pulling Modes in Analog Frequency Dividers
}

\author{
Antonio Buonomo and Alessandro Lo Schiavo \\ Seconda Università degli Studi di Napoli, Via Roma 29, 81031 Aversa, Italy \\ Correspondence should be addressed to Antonio Buonomo; antonio.buonomo@unina2.it
}

Received 3 April 2013; Accepted 10 July 2013

Academic Editor: Mohamad Sawan

Copyright (C) 2013 A. Buonomo and A. Lo Schiavo. This is an open access article distributed under the Creative Commons Attribution License, which permits unrestricted use, distribution, and reproduction in any medium, provided the original work is properly cited.

\begin{abstract}
We compare the main analytical results available to estimate the locking range, which is the key figure-of-merit of $L C$ frequency dividers based on the injection locking phenomenon. Starting from the classical result by Adler concerning injection-locked oscillators, we elucidate the merits and the shortcomings of the different approaches to study injection-locked frequency dividers, with particular emphasis on divider-by-2. In particular, we show the potential of a perturbation approach which enables a more complete analysis of frequency dividers, making it possible to calculate not only the amplitude and the phase of the locked oscillation, but also the region where it exists and is stable, which defines the locking region. Finally, we analyze the dynamical behaviour of the dividers in the vicinity of the boundary of the locking region, showing that there exists a border region where the occurrence of the locking or the pulling operation mode is possible, depending on the initial conditions of the system.
\end{abstract}

\section{Introduction}

The phenomenon of injection locking [1], or frequency entrainment, of an oscillator through an external signal underlies the operation of injection-locked frequency dividers (ILFDs), which are nowadays realized on-chip, in a number of ways suited for RF integrated circuits. The self-oscillation that characterizes the operation of ILFDs is responsible for the low power consumption, which makes them a valuable alternative to digital frequency dividers, and to Miller's type dividers, in high-frequency low-power applications.

The first, and more known, formulations of the injection locking phenomenon date back to Van der Pol and to a bright intuition of Adler, who obtained results very useful for applications by using a pragmatic approach in the study of a particular vacuum tube oscillator [2]. This phenomenon has recently been considered in a number of papers aimed at obtaining useful design guidelines for analog integrated dividers based on the injection locking of $L C$ differential oscillators in MOS technology [3-13]. An ILFD operates properly as a frequency divider only if the basic $L C$ oscillator tracks the input signal, and this occurs over a limited range of frequency, called locking range $(L R)$; its prediction is the main issue of investigation. Predicting the $L R$ can be accurately performed through numerical simulations [14], but analytical methods based on approximate models are able to provide a better insight into the synchronization mechanism which is useful for their design. As a lot of formulas for predicting the locking range as a function of the circuit parameters have been presented in the literature, this often creates uncertainty in their use, especially if the validity limits are not clear.

For the aforementioned reasons, first we present a tight comparison among the main analytical results available for the $L R$, highlighting for each of them the conditions under which they were derived, thus, simplifying the comparison and clarifying their applicability. Starting from the equivalent circuit usually employed for describing the $L C$ frequency dividers and the classical result by Adler concerning injection-locked oscillators, we present the models of ILFDs with saturation-like nonlinearities and with polynomiallike nonlinearities. Then, we show the potential of the perturbation approach in [12], which provides analytical results more complete than those in [5-11], as it results from an analytical and numerical comparison. The perturbation 
approach allows us to determine not only the amplitude and phase of the locked oscillation and the locking range, but also the stability of the oscillation and its envelope in transient. Finally, we study the dynamical behaviour of the dividers in the vicinity of the boundary of the locking region, showing that there exists a border region where the occurrence of the locking or the pulling operation mode is possible, depending on the initial conditions. Numerical simulations are presented to validate the presented analytical results.

\section{Equivalent Circuit of ILFDs}

In order to simplify the study of ILFDs aimed at obtaining simple formulas relating the $L R$ to the circuit parameters, the actual ILFDs are usually reduced to a more simple equivalent circuit. Taking into account that ILFDs are based on a basic injection-locked $L C$ oscillator, it is natural to represent them through the equivalent circuit shown in Figure 1. This can represent an injection-locked $L C$ oscillator, if the external forcing signal, $v_{\text {in }}$, has a frequency close to the tank resonant frequency, while it can represent an injection-locked frequency divider if the external signal has a frequency close to an integer multiple of the tank resonant frequency.

The transfer function of the tank in Figure 1 is

$$
\begin{aligned}
H(j \omega) & =\frac{H_{0}}{1+j Q\left(\left(\omega^{2}-\omega_{0}^{2}\right) / \omega_{0} \omega\right)} \\
& \approx \frac{H_{0}}{1+j\left(2 Q / \omega_{0}\right)\left(\omega-\omega_{0}\right)},
\end{aligned}
$$

where $H_{0}=R$ denotes the tank losses, $\omega_{0}=1 / \sqrt{L C}$ denotes the tank resonant frequency, and $Q=R \sqrt{C / L}$ denotes its quality factor. The nonlinear two-terminal connected to the $L C$ tank in Figure 1 is represented by the function $i=i\left(v, v_{\text {in }}\right)$, where $v$ denotes the tank voltage and $v_{\text {in }}=V_{\text {in }} \cos (n \omega t)$ denotes the input voltage, which is assumed to be purely sinusoidal with a frequency $\omega$ close to an integer multiple $n$ of $\omega_{0}$. In the case here treated of divide-by-2 ILFDs, we have $n=2$. Thus, the circuit in Figure 1 can be modeled by the nonlinear differential equation

$$
\frac{d^{2} v}{d t^{2}}+\frac{\omega_{0}}{Q} \frac{d v}{d t}+\omega_{0}^{2} v=-\frac{\omega_{0} R}{Q} \frac{d}{d t} i\left(v, v_{\text {in }}\right)
$$

Further approximations can be very useful to simplify the study of the equivalent circuit shown in Figure 1 and described by (2). The $L C$ tank is usually assumed to filter out all of the harmonics of the current $i=i\left(v, v_{\text {in }}\right)$ injected into the $L C$ tank, so that the output voltage can be assumed to be purely sinusoidal, $v=V \cos (\omega t+\theta)$, and the amplitude $V_{\text {in }}$ of the injection signal is assumed to be sufficiently small. Moreover, the nonlinear function $i=i\left(v, v_{\text {in }}\right)$ is approximated by a saturation function or by a polynomial function depending on the topology of the actual circuit. In the following sections, we will treat separately these two cases, and we will derive analytical expressions of the $L R$ as a function of the circuit parameters.

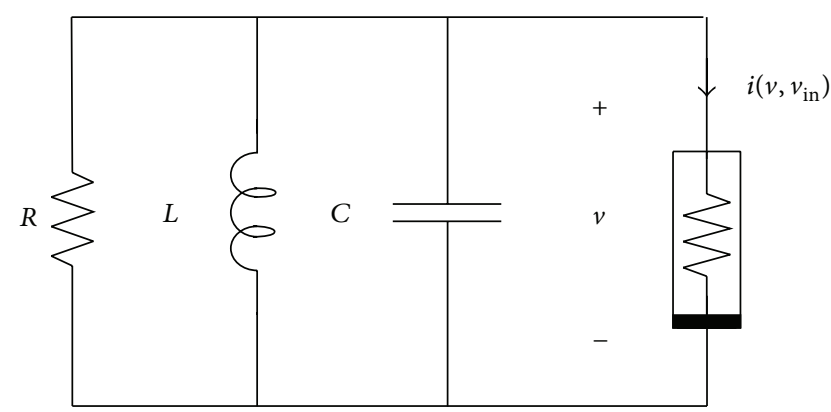

Figure 1: Equivalent circuit of an injection-locked frequency divider.

\section{Analysis of ILFDs}

Different approaches are available in the literature to derive analytical expressions of the $L R$ as a function of the circuit parameters. The most widely used are presented here first by considering the case of active nonlinearities of saturation type and then the case of nonlinearities of polynomial type.

3.1. Saturation-Like Nonlinearity. Here, we investigate the behaviour of the equivalent circuit shown in Figure 1, assuming that the nonlinearity of the active part of the circuit, $i=i\left(v, v_{\text {in }}\right)$, can be represented by a saturation function. This model describes the well-known topology of ILFDs based on two cross-coupled MOS devices with a tail device, through which the injection signal is applied $[9,12]$.

We start our investigation from the classical approach of Adler to study injection-locked oscillators because Adler's results can be easily extended to injection-locked frequency dividers, as we will show. The injection-locked oscillator analyzed in [2] by Adler can be described by the equivalent circuit in Figure 1, wherein the nonlinearity is of saturation type; that is, $i\left(v, v_{\text {in }}\right)=-I_{0} \operatorname{sgn}\left(v+v_{\text {in }}\right)$ with $v_{\text {in }}=V_{\text {in }} \cos (\omega t)$ and $\omega$ close to $\omega_{0}$. It is assumed that the injection amplitude $V_{\text {in }}$ is very small and the amplitude $V$ of the output voltage $v=V \cos (\omega t+\theta)$ does not depend on the amplitude and frequency of the injection signal.

Exploiting the assumption of very small injection amplitude, the expression of the input voltage of the nonlinearity $v_{\mathrm{nl}}=v+v_{\text {in }}$ can be written as

$$
\begin{aligned}
v_{\mathrm{nl}} & =\left[V+V_{\mathrm{in}} \cos \theta\right] \cos (\omega t+\theta)+V_{\mathrm{in}} \sin \theta \sin (\omega t+\theta) \\
& \approx V \cos (\omega t+\theta)+V_{\mathrm{in}} \sin \theta \sin (\omega t+\theta) .
\end{aligned}
$$

As the nonlinearity is memoryless, the phase of the first harmonic of $i$, that is, $i^{(1)}$, is equal to that of $v_{\mathrm{nl}}$, that is, $\angle i^{(1)}=$ $\arctan \left(V_{\text {in }} \sin \theta / V\right)$, while the amplitude is constant due to the saturation nonlinearity. As the instantaneous frequency is $\omega+\dot{\theta}$, the phase shift due to the tank is equal to $\angle H(j(\omega+\dot{\theta}))=$ $-\arctan \left[2 Q\left(\omega+\dot{\theta}-\omega_{0}\right) / \omega_{0}\right]$. Imposing the current-voltage phase relationship $\angle H(j(\omega+\dot{\theta}))-\angle i^{(1)}=\angle v$, a dynamic 
equation for the phase of the output voltage, the so-called Adler equation, is obtained by

$$
\dot{\theta}=\Delta-\frac{\omega_{0}}{2 Q} \frac{V_{\text {in }}}{V} \sin \theta,
$$

where $\Delta=\omega_{0}-\omega$ represents the frequency detuning. Considering that in steady state $\dot{\theta}=0$ and $\sin \theta$ lies between +1 and -1 , we can calculate the maximum and minimum possible values of $\Delta$, that is,

$$
\Delta_{M}= \pm \frac{\omega_{0}}{2 Q} \frac{V_{\text {in }}}{V}
$$

which define the boundaries of the $L R$, whose extension is $L R=2|\Delta|_{M}$.

An expression similar to (5) is obtained in [5] for a current driven oscillator. Imposing $i\left(v, v_{\text {in }}\right)=i_{\text {nl }}(v)+I_{\text {in }} \cos (\omega t)$, where $i_{\mathrm{nl}}(v)$ is the oscillator nonlinearity, the perturbation projection vector (PPV) of the oscillator is analytically calculated and we obtain $L R=\left(\omega_{0} / Q\right)\left(I_{\text {in }} / I_{R}\right)$, where $I_{R}$ is the current in the loss resistance.

The approach proposed by Paciorek [6] is equal to that in [2], but it holds for injection amplitudes larger than those considered in [2]. Repeating the same developments shown before without making the approximation in (3), we get

$$
\Delta_{M}= \pm \frac{\omega_{0}}{2 Q} \frac{V_{\text {in }}}{V} \frac{1}{\sqrt{1-\left(V_{\text {in }} / V\right)^{2}}} .
$$

An expression similar to (6) for an $L C$ tank with a current injection, instead of a voltage injection, is obtained in [7] using a graphical steady-state approach and in [8] by resorting a complex low-pass filter equivalence. Imposing $i\left(v, v_{\text {in }}\right)=i_{\text {nl }}(v)+I_{\text {in }} \cos (\omega t)$, we obtain an expression similar to that in (6), wherein $I_{\text {in }} / I_{\mathrm{nl}}$ is in place of $V_{\text {in }} / V$, with $I_{\mathrm{nl}}$ denoting the amplitude of the current that is fed back by the limiting amplitude nonlinearity $i_{\text {nl }}(v)$.

The presented approaches to study injection-locked oscillators can be extended to analyze frequency dividers. A divide-by-2 ILFD based on tail injection can be described by the circuit in Figure 1, where the saturation nonlinearity is slightly different from that considered in [2]; that is, $i\left(v, v_{\text {in }}\right)=$ $-I_{0} \operatorname{sgn}(v)\left(1+k v_{\text {in }}\right)$ with $v=V \cos (\omega t+\theta), v_{\text {in }}=V_{\text {in }} \cos (2 \omega t)$, and $\omega$ close to $\omega_{0}$.

In line with the approach in [2] and assuming a very small injection amplitude, the following approximation is possible for the first harmonic of the current:

$$
\begin{aligned}
i^{(1)}= & -\left(\frac{4 I_{0}}{\pi}+\frac{4 I_{0}}{3 \pi} k V_{\text {in }} \cos 2 \theta\right) \cos (\omega t+\theta) \\
& -\frac{8 I_{0}}{3 \pi} k V_{\text {in }} \sin 2 \theta \sin (\omega t+\theta) \\
\approx & -\frac{4 I_{0}}{\pi} \cos (\omega t+\theta)-\frac{8 I_{0}}{3 \pi} k V_{\text {in }} \sin 2 \theta \sin (\omega t+\theta) .
\end{aligned}
$$

Then, imposing the current-voltage phase relationship, a dynamic equation for the phase of the output voltage is obtained as follows:

$$
\dot{\theta}=\Delta-\frac{\omega_{0}}{Q} \frac{k V_{\text {in }}}{3} \sin \theta .
$$

From (8), the expressions of the boundaries of the $L R$ are derived by

$$
\Delta_{M_{-} \mathrm{AD}}= \pm \frac{\omega_{0}}{\mathrm{Q}} \frac{k V_{\text {in }}}{3},
$$

for an ILFD with saturation-like characteristic. Equation (9), which is valid for an ILFD, was obtained using the same approach used by Adler to study an injection-locked oscillator and represents a reference for every study on the locking range of ILFDs.

On the other hand, if the simplifying approximation in (7) is not made in line with the procedure presented in [6], we get

$$
\Delta_{M \_P A}= \pm \frac{\left(\omega_{0} / Q\right)\left(k V_{\text {in }} / 3\right)}{\sqrt{1-\left(k V_{\text {in }} / 3\right)^{2}}} .
$$

In the following section, we show that (9) provides an estimate of the $L R$ that is smaller than the actual one, while (10) provides an estimate larger than the actual $L R$.

In line with Verma-Rategh-Lee's approach [9], ILFDs are studied approximating the nonlinear function $i=f\left(v, v_{\text {in }}\right)$ in the form $i\left(v, v_{\text {in }}\right)=i_{\text {nl }}(v)+g(v) v_{\text {in }}$ with $i_{\text {nl }}(v)=i(v, 0)$ and $g(v)=\partial i / \partial v_{\text {in }}$. Then, applying the steady-state Barkhausen criterion and assuming that the oscillation amplitude $V$ does not depend on the frequency of the injection signal, we obtain

$$
\Delta_{M}=\frac{\omega_{0}}{2 Q} \arctan \frac{\left|k_{+}-k_{-}\right|}{\sqrt{1-\left(k_{+}+k_{-}\right)^{2}}},
$$

where $k_{ \pm}=V_{\text {in }} G_{2 \pm 1} /\left(2 I_{\mathrm{nl}}\right), I_{\mathrm{nl}}$ is the cosine Fourier coefficient of the first harmonic of $i_{\mathrm{nl}}(V \cos (\omega t+\theta))$, and $G_{2 \pm 1}$ is the cosine Fourier coefficient of the harmonic of order $2 \pm 1$ of $g(V \cos (\omega t+\theta))$. Particularizing (11) for the case of saturationlike nonlinearity, that is, $i\left(v, v_{\text {in }}\right)=-I_{0}\left(1+k v_{\text {in }}\right) \operatorname{sgn}(v)$, for which $i(v, 0)=-I_{0} \operatorname{sgn}(v), g(v)=-k I_{0} \operatorname{sgn}(v), I_{\mathrm{nl}}=-4 I_{0} / \pi$, $G_{1}=I_{n l}, G_{3}=-G_{1} / 3, k_{-}=V_{\text {in }} k / 2$, and $k_{+}=-k_{-} / 3$, we obtain

$$
\Delta_{M_{-} V R L}= \pm \frac{\omega_{0}}{2 Q} \arctan \frac{2 k V_{\text {in }}}{3} .
$$

It is interesting to observe that in case of small injection amplitudes, the arctan function can be approximated by its argument and, hence, (12) is reduced to (9).

3.2. Polynomial-Like Nonlinearity. Let us now consider the case that the nonlinearity of the current source in Figure 1 is represented by a polynomial function. This is the case of injection-locked Colpitts oscillators [10], of ILFDs with feedthrough, and of CMOS differential ILFDs with direct injection $[12,13]$.

The model used in [10] for a divide-by-2 Colpitts ILFD is shown in Figure 1 with a nonlinearity of cubic type; that is, $i=a_{0}+a_{1}\left(v+v_{\text {in }}\right)+a_{2}\left(v+v_{\text {in }}\right)^{2}+a_{3}\left(v+v_{\text {in }}\right)^{3}$. Considering that the complex expression of the first harmonic of $i$ is $I=I^{c}+j I^{s}$ with

$$
\begin{gathered}
I^{c}=a_{1} V+\frac{3}{4} a_{3} V^{3}+\frac{3}{2} a_{3} V V_{\text {in }}^{2}+a_{2} V V_{\text {in }} \cos (2 \theta), \\
I^{s}=a_{2} V V_{\text {in }} \sin (2 \theta),
\end{gathered}
$$


and applying the steady-state Barkhausen criterion $\left(I^{c}+\right.$ $\left.j I^{s}\right) H_{0}=V\left(1+j 2 Q\left(\omega-\omega_{0}\right) / \omega_{0}\right)$, the balance of the imaginary components leads to the following:

$$
\Delta_{M \_R L}= \pm \frac{\omega_{0}^{2} L\left|a_{2}\right| V_{\text {in }}}{2}
$$

which is obtained when considering that $\sin (2 \theta)$ lies between +1 and -1 .

It is interesting to observe that this expression is significantly different from those in (9) and (10), as it shows no dependence on the tank losses. Thus, in this case the $L R$ can be widened without lowering the tank quality factor and, hence, without increasing the phase noise of the output signal.

Polynomial nonlinearity is also used in [11]. The equivalent circuit shown in Figure 1 with a cubic feedback nonlinearity models an ILFD based on a mixer, wherein the fed-back output signal does feedthrough to the output of the mixer. In this case, the nonlinear function is as follows:

$$
i\left(v, v_{\text {in }}\right)=-\left[V_{\mathrm{dc}}+V_{\text {in }} \cos (2 \omega \mathrm{t})\right]\left(a_{1} v-a_{3} v^{3}\right),
$$

where $V_{\mathrm{dc}}$ is a measure of the output feedthrough. Applying the stroboscopic method, a first-order system of ordinary differential equations in the amplitude and phase variables is derived. Even if the resulting system cannot be solved in closed form, by using simplifying assumptions it is possible to obtain an estimate of the locking interval, that is,

$$
\Delta_{M \_ \text {SBH }}= \pm \frac{\omega_{0}^{2} L a_{1} V_{\text {in }}}{4}\left[1-\frac{2}{3} \frac{\left(a_{1} R V_{\mathrm{dc}}-1\right)}{a_{1} R V_{\mathrm{dc}}}\right] \text {, }
$$

in the assumption that $a_{1} R V_{\mathrm{dc}}>1$. It is interesting to observe that $a_{1} R V_{\mathrm{dc}}=1$ is the limit condition that ensures a sustained oscillation in absence of the input signal, and it is also the condition that provides the maximum locking range.

\section{The Perturbation Approach as Compared to Previous Approaches}

Injection-locked frequency dividers are analyzed in [12] by considering that they are made of a basic LC oscillator, whose active part is modelled by $i_{\mathrm{nl}}(v)$, and of an injection circuit modelled by the nonlinearity $i_{\text {in }}\left(v, v_{\text {in }}\right)$. Thus, they can be modelled by the circuit in Figure 1 with $i\left(v, v_{\text {in }}\right)=$ $i_{\text {nl }}(v)+i_{\text {in }}\left(v, v_{\text {in }}\right)$. Then, the averaging equations are obtained by approximating the nonlinearity in the form $i\left(v, v_{\text {in }}\right)=$ $i_{\text {nl }}(v)+i_{\text {in }}(v, 0)+g(v) v_{\text {in }}$ where $g(v)=\partial i / \partial v_{\text {in }}$ and by applying a perturbation method. It results in the following:

$$
\begin{aligned}
\dot{V}(t)= & -\frac{\omega_{0}}{2 Q} \\
& \times\left[V+R\left(I_{\mathrm{nl}}^{c}+I_{\mathrm{in} 0}^{c}+\frac{V_{\mathrm{in}}}{2}\left(G_{2-1}+G_{2+1}\right) \cos (2 \theta)\right)\right], \\
& \dot{\theta}(t)=\Delta+\frac{\omega_{0} R V_{\mathrm{in}}}{4 Q V}\left(G_{2-1}-G_{2+1}\right) \sin (2 \theta),
\end{aligned}
$$

where $\Delta$ denotes the frequency detuning $\omega_{0}-\omega, G_{k}$ denote the Fourier coefficients of the function $g(V \cos (\omega t+\theta))$, and $I_{\mathrm{nl}}^{\mathrm{c}}$ and $I_{\mathrm{in} 0}^{\mathrm{c}}$ denote the first-harmonic coefficients of $i_{\mathrm{nl}}(v)$ and of $i_{\text {in }}(v, 0)$, respectively.

First-order dynamical system (17), which describes the frequency dividers in terms of amplitude and phase, allows us to determine not only the amplitude and phase in steady state, and the locking range, but also the stability of the oscillation and its envelope in transient. It should be highlighted that (17) allows us to take into account the dependence of $V$ on the frequency, differently from $[2,9]$. Moreover, it is interesting to observe that it is not sufficient to determine if a solution of (17) does exist, as made in $[2,6,9,10]$, but it is also necessary to establish if it is stable and, thus, observable in practice.

However, even if the model (17) is very comprehensive and powerful, it is not exactly equivalent to that described by the original nonlinear differential equation in (2) due to the assumption of negligible harmonics on the tank voltage. However, the latter is usually verified by the circuit parameters of practical ILFDs.

Here, we show that the presented approach allows us to obtain results more accurate than those presented in Section 3.1 for the locking range of ILFDs with saturationlike nonlinearities. Moreover, it allows us to obtain the same results presented in Section 3.2 for the locking range of ILFD with polynomial-like nonlinearities, but it enables us also to obtain the amplitude and the phase of the oscillation.

4.1. Saturation-Like Nonlinearity. If the active part of the frequency divider can be modelled by a saturation-like nonlinearity [15-17], that is, $i\left(v, v_{\text {in }}\right)=-I_{0}\left(1+k v_{\text {in }}\right) \operatorname{sign}(v)$, the following coefficients are obtained for the harmonics $I_{\mathrm{nl}}^{c}=$ $-4 I_{0} / \pi, I_{\mathrm{in} 0}^{c}=0, G_{1}=k_{p} I_{\mathrm{nl}}^{c}$, and $G_{3}=-G_{1} / 3$, and model (17) leads to the following:

$$
\begin{gathered}
\dot{V}(t)=-\frac{\omega_{0}}{2 Q}\left[V-V_{s s}\left(1+\frac{k V_{\text {in }}}{3} \cos 2 \theta\right)\right], \\
\dot{\theta}(t)=\Delta-\frac{V_{s s}}{V} \frac{\omega_{0}}{Q} \frac{k V_{\text {in }}}{3} \sin 2 \theta,
\end{gathered}
$$

with $V_{s s}=4 R I_{0} / \pi$. It should be highlighted that system (18) is more general than the models presented in $[2,6,9]$. Actually, if the output amplitude $V$ is assumed to be constant, system (18) leads to a Paciorek-like equation. If the output amplitude $V$ is assumed to be constant and equal to the amplitude in free-running mode of operation, that is, $V=V_{s s}$, then system (18) leads to the Adler-like equation (8). Finally, if both the output amplitude $V$ and the phase $\theta$ are assumed to be constant, system (18) leads to the Barkhausen equations in [9].

The steady-state solution of (18) is as follows:

$$
V=\frac{V_{s s}}{1+\Delta^{2} Q^{2} / \omega_{0}^{2}}\left(1+\frac{k V_{\text {in }}}{3} \sqrt{1-\left(\frac{9}{k^{2} V_{\text {in }}^{2}}-1\right) \frac{Q^{2}}{\omega_{0}^{2}} \Delta^{2}}\right),
$$




$$
\theta= \begin{cases}\frac{1}{2} \arcsin \left(\frac{3 \Delta Q V}{\omega_{0} k V_{s s} V_{\text {in }}}\right)+r \pi, & V \geq V_{s s}, \\ \frac{\pi}{2}-\frac{1}{2} \arcsin \left(\frac{3 \Delta Q V}{\omega_{0} k V_{s s} V_{\text {in }}}\right)+r \pi, & V<V_{s s} .\end{cases}
$$

From (19), we deduce that the steady-state solution of (18) does exist in the frequency range $|\Delta| \leq \Delta_{\text {EXIS }}$, where

$$
\Delta_{\text {EXIS }}=\frac{\left(\omega_{0} / \mathrm{Q}\right)\left(k V_{\text {in }} / 3\right)}{\sqrt{1-\left(k V_{\text {in }} / 3\right)^{2}}} .
$$

In order to determine the stability properties of the solution (19), we calculate the eigenvalues of the system obtained by the linearization of (18), obtaining the following:

$$
\lambda_{a, b}=\frac{\operatorname{tr}(M)}{2} \pm \frac{1}{2} \sqrt{\operatorname{tr}(M)^{2}-4 \operatorname{det}(M)},
$$

where $M$ is the Jacobian matrix, whose trace is $\operatorname{tr}(M)=$ $-5 / 2+2 V_{s s} / V$, and whose determinant is $\operatorname{det}(M)=1+$ $\left(Q \Delta / \omega_{0}\right)^{2}-V_{s s} / V$. By evaluating the sign of the quantities in (21), we deduce the interval wherein the solution is stable by

$$
\Delta_{M_{-} \mathrm{BL}}= \begin{cases} \pm \Delta_{\mathrm{EXIS}}, & 0<\frac{k V_{\text {in }}}{3}<\frac{1}{\sqrt{5}}, \\ \pm \frac{\omega_{0}}{4 Q} \sqrt{25\left(\frac{k V_{\text {in }}}{3}\right)^{2}-1}, & \frac{1}{\sqrt{5}}<\frac{k V_{\text {in }}}{3}<1 .\end{cases}
$$

It is interesting to observe that the frequency range in (20) is equal to the $L R$ obtained by the Paciorek-like approach in (10), but the approach in [12] allows us to establish that the range with locked oscillations is stable and, thus, can be observed; that is, the $L R$ is smaller than that in (20) and is equal to that in (22).

In order to simplify the comparison among the formulas that predict the locking range of dividers with saturationlike nonlinearity, that is, (9), (10), (12), and (22), it is useful to normalize the boundaries of the locking range $\Delta_{M}$, that is, $\Delta_{M n}=\Delta_{M} \mathrm{Q} / \omega_{0}$, and the injection amplitude, $\mathrm{V}_{\text {in }}$, that is, $m=k V_{\text {in }} / 3$. Figure 2, where the results obtained by formulas and by SPICE simulations are reported, shows that the considered formulas are equivalent for small injection values; that is, $m<0.2$. By increasing the injection amplitude, the $L R$ s in (9) and (12) are significantly smaller than the $L R$ predicted by (22), while the $L R$ in (10) is wider. Comparing these values with those obtained by numerical simulations, we deduce that the best estimation of the $L R$ is provided by (22). Moreover, we observe that the boundaries of the $L R$ obtained by numerical simulations are not symmetric with respect to $\Delta=0$, but are different from the boundaries obtained by formulas, because they do not take into account the presence of harmonics. In order to further compare the results from analytical expressions of the $L R$ and the results from SPICE simulations, in Figure 3 we show the results obtained for different values of the parameters of the passive and active components. Results in Figure 3 confirm that the best analytical estimation of the $L R$ is provided by (22).

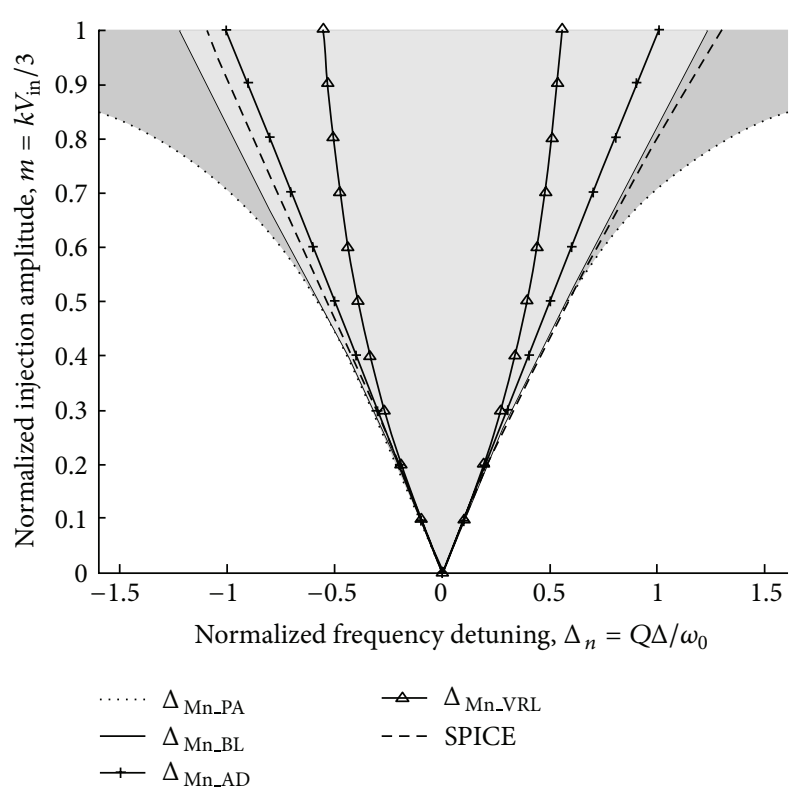

Figure 2: Comparison of the boundaries of the locking range (Arnold's tongue) for a frequency divider with saturation-like nonlinearity, obtained by formulas and by numerical simulations. $\Delta_{M_{-} \mathrm{PA}}$ is shown in (10), $\Delta_{M_{-} \mathrm{BL}}$ in (22), $\Delta_{M_{-} \mathrm{AD}}$ in (9), and $\Delta_{M_{-} V R L}$ in (12). Numerical simulations are obtained by using the following parameters of the circuit in Figure 1: $L=4 \mathrm{nH}, R=400, \omega_{0}=$ $1.8 \mathrm{GHz}, I_{0}=2 \mathrm{~mA}$, and $k=2.6 / \mathrm{V}$.

4.2. Polynomial-Like Nonlinearity. The approach in [12] allows us also to determine the locking range of ILFDs in case of polynomial nonlinearity. Let us first consider the case that the nonlinearity can be written as $i=a_{1} v+a_{3} v^{3}+b|v| v+k v v_{\text {in }}$. This model applies, as an example, to CMOS ILFDs with direct injection [12, 18-20]. In this case, the averaging system (17) is reduced to the following:

$$
\begin{gathered}
\dot{V}(t)=-\frac{\omega_{0} R}{2 Q} V\left(\frac{1}{R}+a_{1}+\frac{8 b}{3 \pi} V+\frac{3}{4} a_{3} V^{2}+\frac{k V_{\text {in }}}{2} \cos 2 \theta\right), \\
\dot{\theta}(t)=\Delta+\frac{\omega_{0}}{4 Q} R k V_{\text {in }} \sin 2 \theta,
\end{gathered}
$$

whose steady-state solution is

$$
\begin{gathered}
V=\sqrt{-\frac{4}{3} \frac{a_{1} R+1}{a_{3} R}+\left(\frac{16 b}{9 \pi a_{3}}\right)^{2}-\frac{2 k V_{\text {in }}}{3 a_{3}} \sqrt{1-\left(\frac{4 \Delta}{\omega_{0}^{2} L k V_{\text {in }}}\right)^{2}}} \\
-\frac{16 b}{9 \pi a_{3}}, \\
\theta=-\frac{1}{2} \arcsin \left(\frac{4 \Delta}{\omega_{0}^{2} L k V_{\text {in }}}\right)+r \pi .
\end{gathered}
$$

By calculating the frequency range where the solution (24) does exist and it is stable, we deduce the boundaries of the $L R$ as follows:

$$
\Delta_{M}= \pm \omega_{0}^{2} L \frac{|k| V_{\text {in }}}{4}
$$




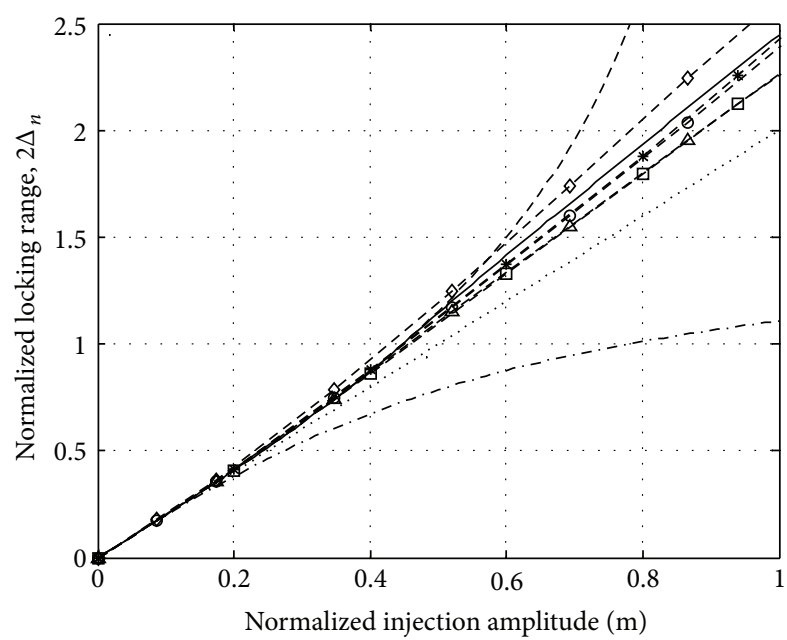

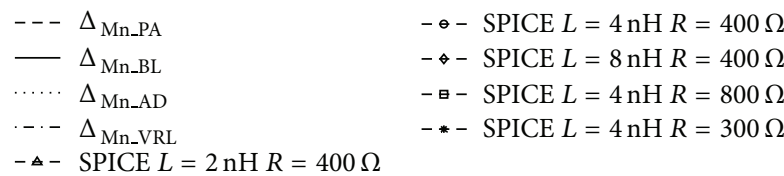

(a)

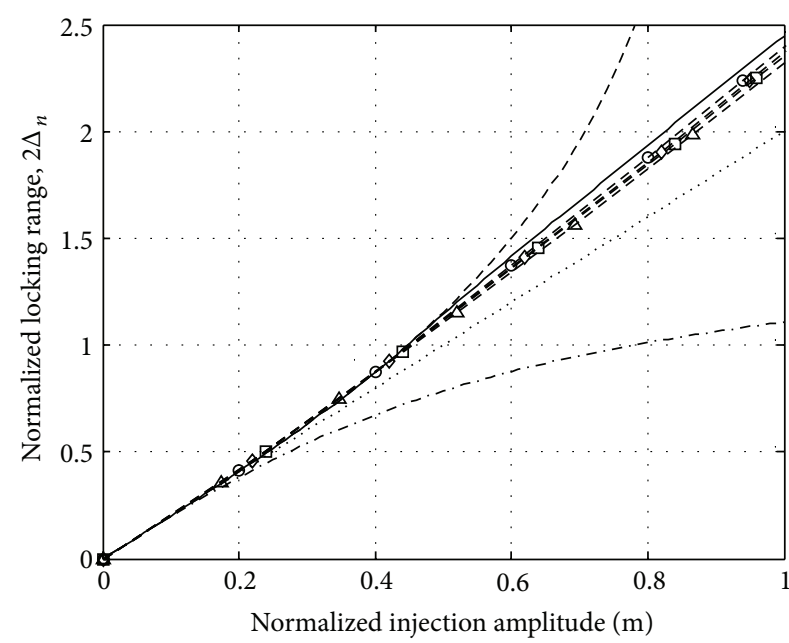

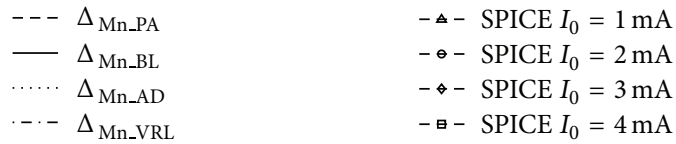

(b)

FIgURE 3: Normalized locking ranges for an ILFD with saturation-like nonlinearity. Parameters of the SPICE simulations of the circuit in Figure 1 are the following: $L=4 \mathrm{nH}$, and $R=400, \omega_{0}=1.8 \mathrm{GHz}, I_{0}=2 \mathrm{~mA}, k=2.6 / V$.

which, differently from the case of ILFD with saturation-like nonlinearity, do not depend on the circuit losses.

The approach in [12] is general enough to treat also the cases of polynomial nonlinearities discussed in Section 3.2. In the case that $i=a_{0}+a_{1}\left(v+v_{\text {in }}\right)+a_{2}\left(v+v_{\text {in }}\right)^{2}+a_{3}\left(v+v_{\text {in }}\right)^{3}$, the steady-state solution of the averaging system (17) gives the following:

$$
\begin{gathered}
V=\sqrt{-\frac{4}{3} \frac{a_{1} R+1}{a_{3} R}-2 V_{\mathrm{in}}^{2}-\frac{4 a_{2} V_{\mathrm{in}}}{3 a_{3}} \sqrt{1-\left(\frac{2 \Delta}{\omega_{0}^{2} L a_{2} V_{\mathrm{in}}}\right)^{2}},} \\
\theta=-\frac{1}{2} \arcsin \left(\frac{2 \Delta}{\omega_{0}^{2} L a_{2} V_{\mathrm{in}}}\right)+r \pi .
\end{gathered}
$$

Calculating the interval of existence of the solution (26) we obtain the same boundaries of the $L R$ reported in (14).

Finally, in the case that the nonlinearity is described by (15), the averaging system (17) is reduced to the following:

$$
\begin{aligned}
\dot{V}(t)= & -\frac{\omega_{0} R}{2 Q} \\
& \times V\left(\frac{1}{R}-a_{1} V_{\mathrm{dc}}+\frac{3}{4} a_{3} V_{\mathrm{dc}} V^{2}-\frac{a_{1 c} V_{\mathrm{in}}}{2} \cos 2 \theta\right), \\
& \dot{\theta}(t)=\Delta-\frac{\omega_{0} R}{4 Q} a_{1 s} V_{\mathrm{in}} \sin 2 \theta,
\end{aligned}
$$

where

$$
a_{1 c}=a_{1}\left(1+\frac{a_{3} V^{2}}{a_{1}}\right), \quad a_{1 s}=a_{1}\left(1-\frac{a_{3} V^{2}}{2 a_{1}}\right) .
$$

Simplifying the system through the following approximations:

$$
a_{1 c} \approx a_{1}\left(1+\frac{a_{3} V_{s s}^{2}}{a_{1}}\right), \quad a_{1 s} \approx a_{1}\left(1-\frac{a_{3} V_{s s}^{2}}{2 a_{1}}\right)
$$

where $V_{s s}=\sqrt{4\left(a_{1} V_{\mathrm{dc}} R-1\right) /\left(3 a_{3} V_{\mathrm{dc}} R\right)}$ is the oscillation amplitude in free-running oscillation, it is possible to obtain the steady-state solution in closed form as follows:

$$
\begin{gathered}
V=\sqrt{\frac{4}{3} \frac{a_{1} R V_{\mathrm{dc}}-1}{a_{3} R V_{\mathrm{dc}}}-\frac{2 a_{1 c} V_{\mathrm{in}}}{3 a_{3} V_{\mathrm{dc}}} \sqrt{1-\left(\frac{4 \Delta}{\omega_{0}^{2} L a_{1 s} V_{\mathrm{in}}}\right)^{2}},} \\
\theta=-\frac{1}{2} \arcsin \left(\frac{4 \Delta}{\omega_{0}^{2} L a_{1 s} V_{\mathrm{in}}}\right)+r \pi .
\end{gathered}
$$

From (30), we deduce that the boundaries of the $L R$ are those reported in (16). It is interesting to observe that, even if the system (27) can be practically handled, only if the assumptions in (29) are made, the results are sufficiently accurate, as shown in Figure 4, where analytical results are compared with numerical results obtained by SPICE simulation of the circuit in Figure 1. 


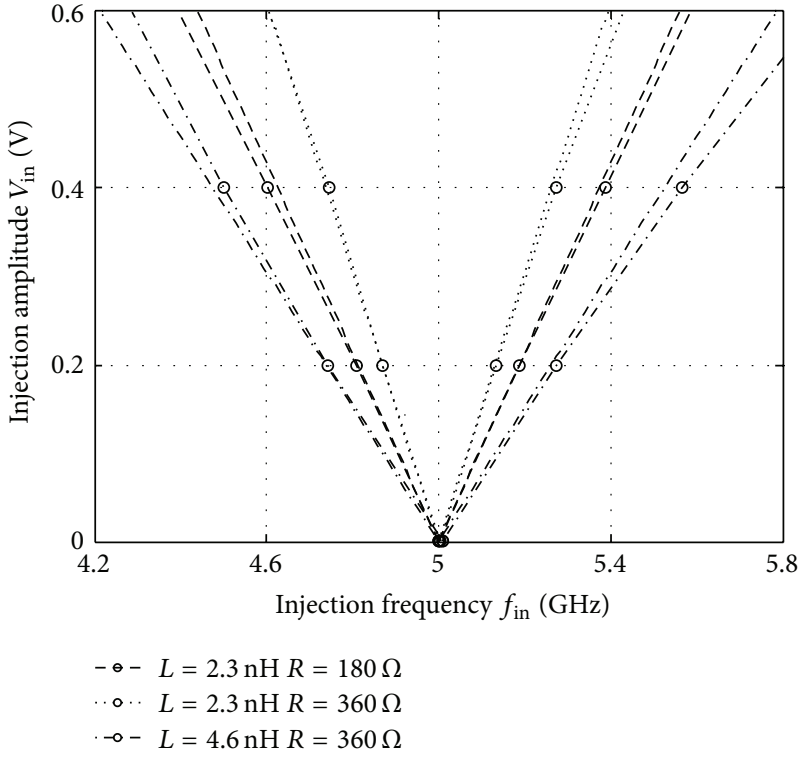

(a)

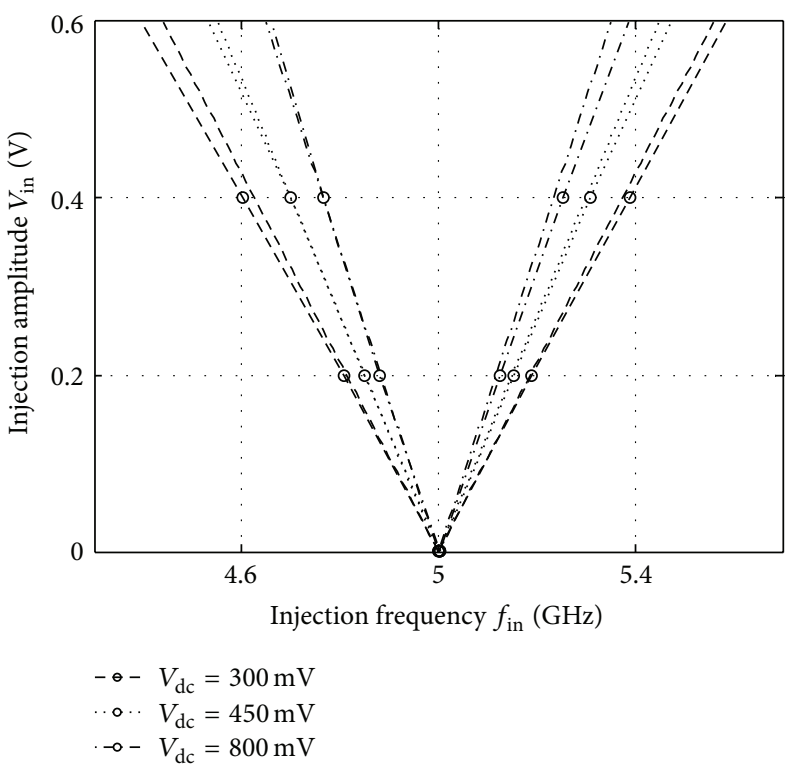

(b)

FIGURE 4: Boundaries of the locking ranges for an ILFD with the polynomial nonlinearity in (15) for different values of passive parameters and feedthrough entity. Results are obtained by SPICE simulations (lines with circles) and by (16) (lines without circles). Circuit parameters are the following: $L=4 \mathrm{nH}, R=400, \omega_{0}=1.8 \mathrm{GHz}, a_{1}=1 / 40, a_{3}=5 / 96$, and $V_{\mathrm{dc}}=300 \mathrm{mV}$.

From the aforementioned analysis, we conclude that the approach in [12] allows us not only to obtain the same results as in $[10,11]$ for the $L R$, but it allows us also to obtain closed form solutions for the amplitude and phase as well as for the stability analysis [13] and the transient behaviour using firstorder dynamical equations.

\section{Operation Modes Near the Boundaries of the Arnold Tongue}

Outside the region which delimits the locked states in Figure 2, as a rule called Arnold's tongue, it is reasonable to expect that the result of the interaction of the oscillator with the forcing signal $v_{\text {in }}$ changes radically, as it happens in an $L C$ oscillator driven by a signal at frequency near to that of the free oscillation $[2,21,22]$. It is known that, near the Arnold tongue, this interaction manifests as a periodically repeated and incomplete frequency entrainment process, known as periodic pulling. This process causes a simultaneous modulation of amplitude and phase of the system's oscillatory response, which has a complex time evolution and exhibits a power spectrum with very dense sidebands. The occurrence of the periodic pulling, of course, should be avoided for a proper operation of the circuit as a frequency divider. In this section, we highlight that the precise location of the boundary between locking and pulling regions, that is, of the bifurcation points from the quasiperiodic states to locked states, is uncertain differently from that predicted by existing analytical models.

The periodic solution in the pulling mode can be found from (18) by putting $V(t)=V_{s s}+\widetilde{v}(t)$ and by making the

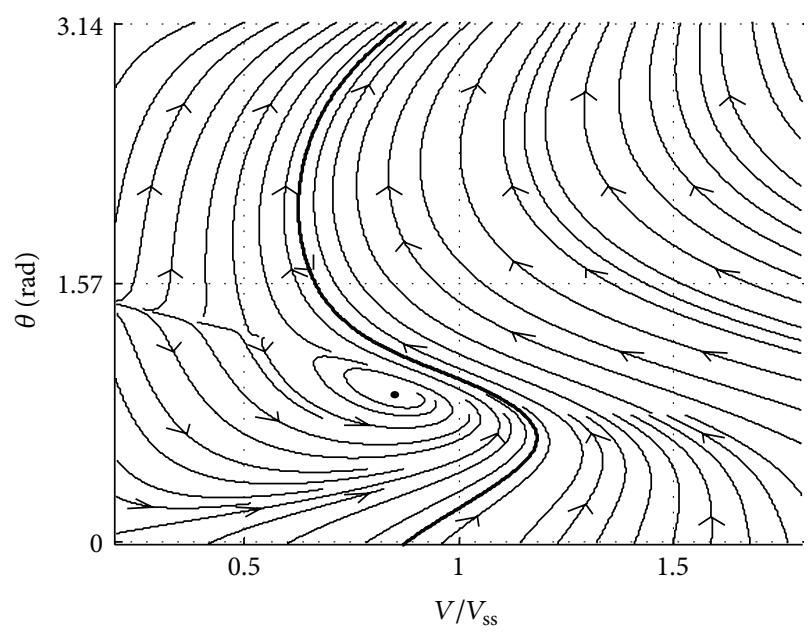

FIGURE 5: Phase portrait of system (18) in case of saturation-like nonlinearity for $k V_{\text {in }} / 3=0.6$ and $Q \Delta / \omega_{0}=0.68$. The black dot denotes a stable steady-state constant solution (locking), while the thick line denotes a stable steady-state periodic solution (pulling).

simplifying assumption $\widetilde{v}(t) \ll V_{s s}$. This reduces (18) to the system of decoupled equations as follows:

$$
\begin{gathered}
\dot{\tilde{v}}(t)=\frac{\omega_{0}}{2 Q} \widetilde{v}+\frac{\omega_{0}}{2 Q} V_{s s} \frac{k_{p} V_{\text {in }}}{3} \cos 2 \theta, \\
\dot{\theta}(t)=\Delta-\frac{\omega_{0}}{Q} \frac{k_{p} V_{\text {in }}}{3} \sin 2 \theta,
\end{gathered}
$$




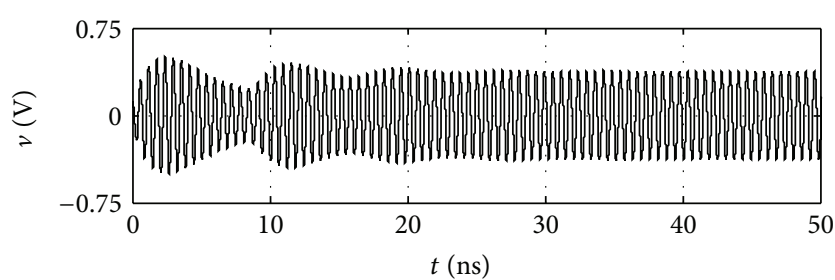

(a)

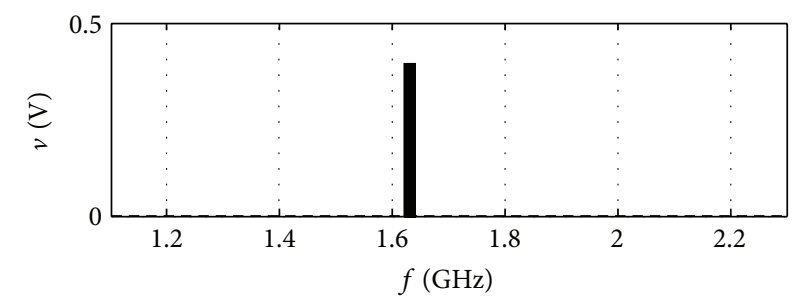

(c)

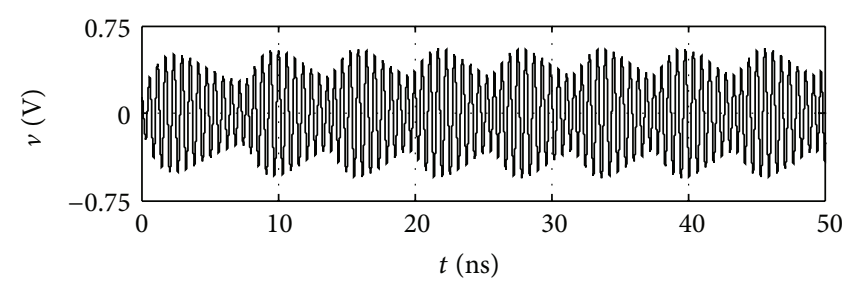

(b)

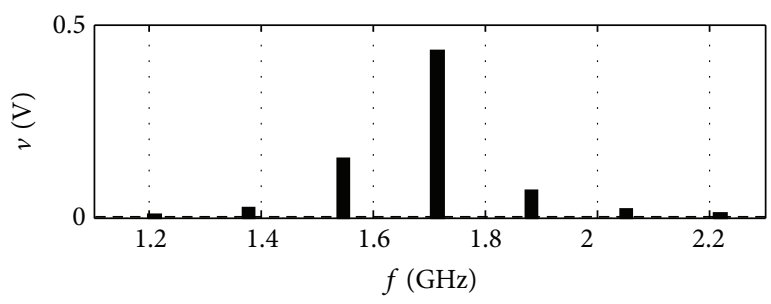

(d)

FIGURE 6: Time waveforms and frequency spectra obtained by SPICE simulations of the circuit in Figure 1 for $L=4 \mathrm{nH}, C=2 \mathrm{pF}, R=400 \Omega$, and $I_{0}=1 \mathrm{~mA}$. Injection amplitude and frequency determine $k V_{\text {in }} / 3=0.6$ and $Q \Delta / \omega_{0}=0.75$. (a) Waveform obtained with initial conditions: $v_{C}(0)=0.1 \mathrm{~V}$ and $i_{L}(0)=1 \mathrm{~mA}$. (b) Waveform obtained with initial conditions: $v_{C}(0)=0.1 \mathrm{~V}$ and $i_{L}(0)=0 \mathrm{~mA}$. (c) Spectrum of the waveform in (a). (d) Spectrum of the waveform in (b).

whose solution can be found in explicit form. According to Adler [2], the periodic solution of (32) is given by

$$
\theta(t)=\tan ^{-1}\left[\frac{1}{K}+\frac{\sqrt{K^{2}-1}}{K} \tan \left(\frac{\Delta \sqrt{K^{2}-1}}{K} t\right)\right],
$$

where $K=Q \Delta /\left(\omega_{0} m\right)$ and $m=k V_{\text {in }} / 3$, which is valid for $|K|<1$, that is, for $|\Delta|>m \omega_{0} / Q$. This latter condition defines the lower bound of the periodic pulling [2]; that is,

$$
\Delta_{\text {pull }}= \pm m \frac{\omega_{0}}{Q} .
$$

The beat frequency $\omega_{p}$ of (33) is $\omega_{p}=2 \Delta \sqrt{1-\left(\Delta_{\text {pull }} / \Delta\right)^{2}}$.

The amplitude modulation $\widetilde{v}$ is obtained by solving the linear equation (31) and knowing the Fourier series expansion for the periodic function $\cos [2 \theta(t)]$. Here, we limit ourselves to observe that this expansion can be derived by calculating the frequency spectrum of $\exp j \theta(t)$ according to the procedure given in [21].

Taking into account that $\Delta_{\text {pull }}$ in (34) is smaller than $\Delta_{M_{\text {_BL }}}$ in (22), it results that the pulling mode of operation and the locking mode of operation coexist in the overlapping intervals $\left|\Delta_{\text {pull }}\right|<\Delta<\left|\Delta_{M_{-} B L}\right|$ and $-\left|\Delta_{M_{B} \text { BL }}\right|<\Delta<-\left|\Delta_{\text {pull }}\right|$.

Numerical integration of system (18) and circuit simulations of the circuit in Figure 1 were performed in order to highlight the behaviour of the circuit near the boundaries of Arnold's tongues. Numerical integration of system (18) allowed us to obtain the phase portrait of system (18) for $k V_{\text {in }} / 3=0.6$ and $Q \Delta / \omega_{0}=0.68$, as reported in Figure 5. Considering the periodicity of the phase $\theta$ over $\pi$, Figure 5 shows that a stable equilibrium point (representative of the locking mode) coexists with a stable equilibrium trajectory (representative of the pulling mode). Thus, the phase portrait confirms the analytical prediction that locking and pulling modes can coexist.

Moreover, SPICE simulations of the circuit in Figure 1 were performed using $k V_{\text {in }} / 3=0.6$ and $Q \Delta / \omega_{0}=0.74$. The waveforms reported in Figures 6(a) and 6(b) clearly show that once the parameter set and the input signal are fixed, the circuit can reach the locking or pulling mode, depending on the initial conditions. Accordingly, when the transient is finished, the spectrum of the steady-state waveforms, shown in Figures 6(c) and 6(d), has a single tone or multiple tones.

Finally, numerical simulations of the circuit in Figure 1 were performed along all of the border of the Arnold's tongue, in order to obtain a complete portrait of the phenomenon. The regions wherein locking and pulling modes coexist, as obtained by numerical simulations and by analytical results, are reported in Figure 7. It shows that both numerical methods and analytical methods predict the presence of a coexistence region, even if there is a slight mismatch between numerical and analytical results due to the effect of harmonics that were neglected in analytical models.

\section{Conclusions}

We showed that the methodology of analysis of ILFDs, based on the well-known asymptotic method of averaging, leads to more general and complete analytical results than those in previous approaches. It allows us to improve the calculation of locking range and to predict the stability of the locked oscillations and their transient behaviour preceding the synchronization state. We investigated in depth the advantages of the method in [12] with respect to previous ones and, at the same time, we highlighted the limits of the analytical approaches in estimating the locking range of ILFDs. The method in [12] allowed us also to analyze the dynamical 


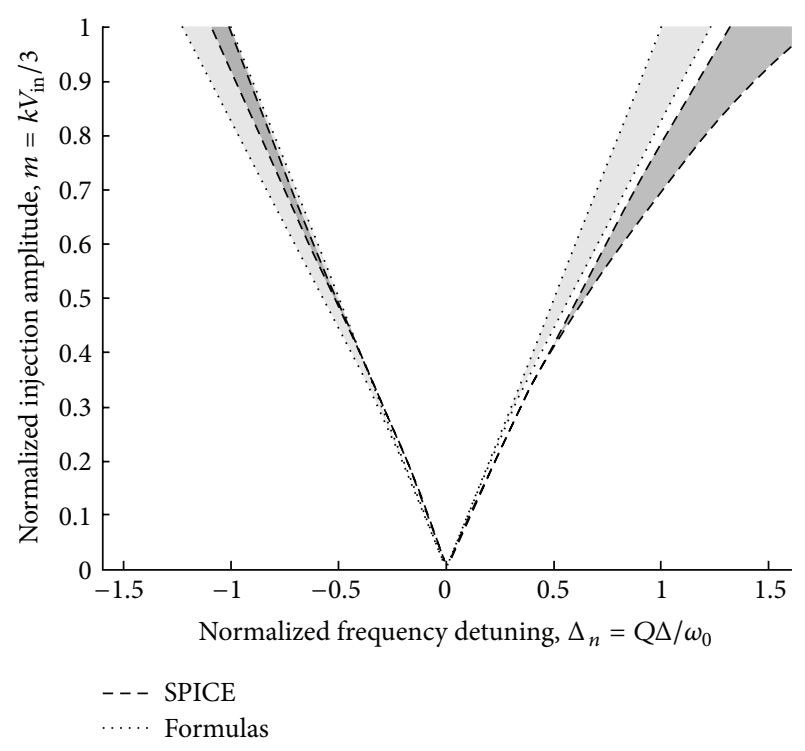

FIGURE 7: Coexistence regions obtained by SPICE simulations (dark gray region) and by analytical formulas (22) and (34) (light gray region).

behavior of the dividers in the vicinity of the boundary of the locking region, showing that there exists a border region where the occurrence of the locking or the pulling operation mode is possible, depending on the initial conditions of the circuit.

\section{References}

[1] K. Kurokawa, "Some basic characteristics of broadband negative resistance oscillator circuits," Bell System Technical Journal, vol. 48, pp. 1937-1955, 1969.

[2] R. Adler, "A study of locking phenomena in oscillators," Proceedings of the IRE, vol. 34, no. 6, pp. 351-357, 1946.

[3] A. Buonomo and A. Lo Schiavo, "Modelling and analysis of differential VCOs," International Journal of Circuit Theory and Applications, vol. 32, no. 3, pp. 117-131, 2004.

[4] A. Buonomo and A. Lo Schiavo, "On the perturbation analysis of the limit cycle in oscillators with shifting bias," International Journal of Circuit Theory and Applications, vol. 28, no. 4, pp. 353$369,2000$.

[5] X. Lai and J. Roychowdhury, "Analytical equations for predicting injection locking in LC and ring oscillators," in Proceedings of IEEE Custom Integrated Circuits Conference (CICC '05), pp. 454-457, September 2005.

[6] L. J. Paciorek, "Injection locking of oscillators," Proceedings of the IEEE, vol. 53, no. 11, pp. 1723-1727, 1965.

[7] B. Razavi, "A study of injection locking and pulling in oscillators," IEEE Journal of Solid-State Circuits, vol. 39, no. 9, pp. 14151424, 2004.

[8] A. Mirzaei, M. E. Heidari, R. Bagheri, S. Chehrazi, and A. A. Abidi, "The quadrature LC oscillator: a complete portrait based on injection locking," IEEE Journal of Solid-State Circuits, vol. 42, no. 9, pp. 1916-1932, 2007.

[9] S. Verma, H. R. Rategh, and T. H. Lee, "A unified model for injection-locked frequency dividers," IEEE Journal of Solid-State Circuits, vol. 38, no. 6, pp. 1015-1027, 2003.
[10] H. R. Rategh and T. H. Lee, "Superharmonic injection-locked frequency dividers," IEEE Journal of Solid-State Circuits, vol. 34, no. 6, pp. 813-821, 1999.

[11] K. Sengupta, T. K. Bhattacharyya, and H. Hashemi, "A nonlinear transient analysis of regenerative frequency dividers," IEEE Transactions on Circuits and Systems I, vol. 54, no. 12, pp. 26462660, 2007.

[12] A. Buonomo and A. Lo Schiavo, "Analytical approach to the study of injection-locked frequency dividers," IEEE Transactions on Circuits and Systems I, vol. 60, no. 1, pp. 51-62, 2013.

[13] A. Buonomo and A. Lo Schiavo, "Nonlinear dynamics of divideby-two injection-locked frequency dividers in locked operation mode," International Journal of Circuit Theory and Applications, 2013.

[14] F. Ramírez, M. E. de Cos, and A. Suárez, "Nonlinear analysis tools for the optimized design of harmonic-injection dividers," IEEE Transactions on Microwave Theory and Techniques, vol. 51, no. 6, pp. 1752-1762, 2003.

[15] A. Ebrahimi, H. Miar Naimi, and H. Adrang, "Remarks on transient amplitude analysis of MOS cross-coupled oscillators," IEICE Transactions on Electronics, vol. 94, no. 2, pp. 231-239, 2011.

[16] A. Buonomo, M. P. Kennedy, and A. Lo Schiavo, "On the synchronization condition for superharmonic coupled QVCOs," IEEE Transactions on Circuits and Systems I, vol. 58, no. 7, pp. 1637-1646, 2011.

[17] A. Buonomo and A. Lo Schiavo, "On the theory of quadrature oscillations obtained through parallel LC-VCOs," IEEE Transactions on Circuits and Systems I, vol. 57, no. 9, pp. 2509-2519, 2010.

[18] S. K. Magierowski and S. Zukotynski, "CMOS LC-oscillator phase-noise analysis using nonlinear models," IEEE Transactions on Circuits and Systems I, vol. 51, no. 4, pp. 664-677, 2004.

[19] A. Buonomo and A. Lo Schiavo, "Modeling, analysis and experimental validation of frequency dividers with direct injection," Journal of Electrical and Computer Engineering, vol. 2013, Article ID 365692, 7 pages, 2013.

[20] A. Buonomo and A. Lo Schiavo, "Finding the tuning curve of a CMOS-LC VCO," IEEE Transactions on Circuits and Systems II, vol. 55, no. 9, pp. 887-891, 2008.

[21] M. Armand, "On the output spectrum of unlocked driven oscillators," Proceedings of the IEEE, vol. 57, no. 5, pp. 798-799, 1969.

[22] I. Ali, A. Banerjee, A. Mukherjee, and B. N. Biswas, "Study of injection locking with amplitude perturbation and its effect on pulling of oscillator," IEEE Transactions on Circuits and Systems I, vol. 59, no. 1, pp. 137-147, 2012. 

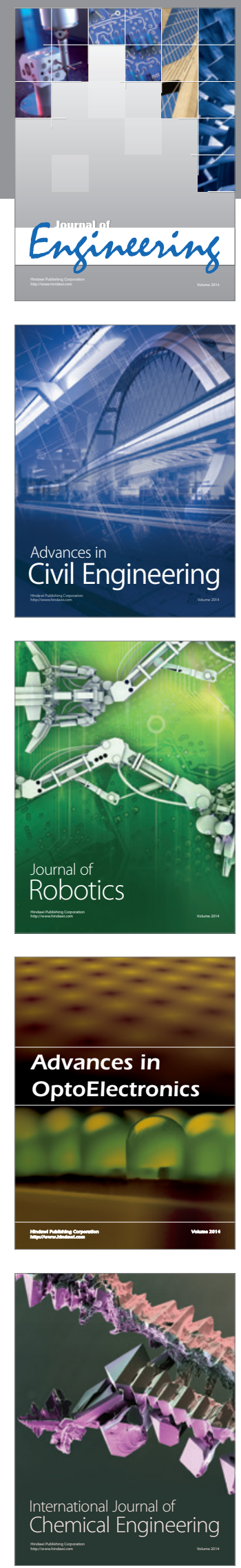

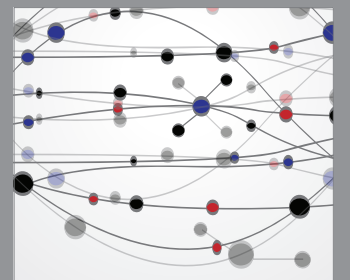

The Scientific World Journal
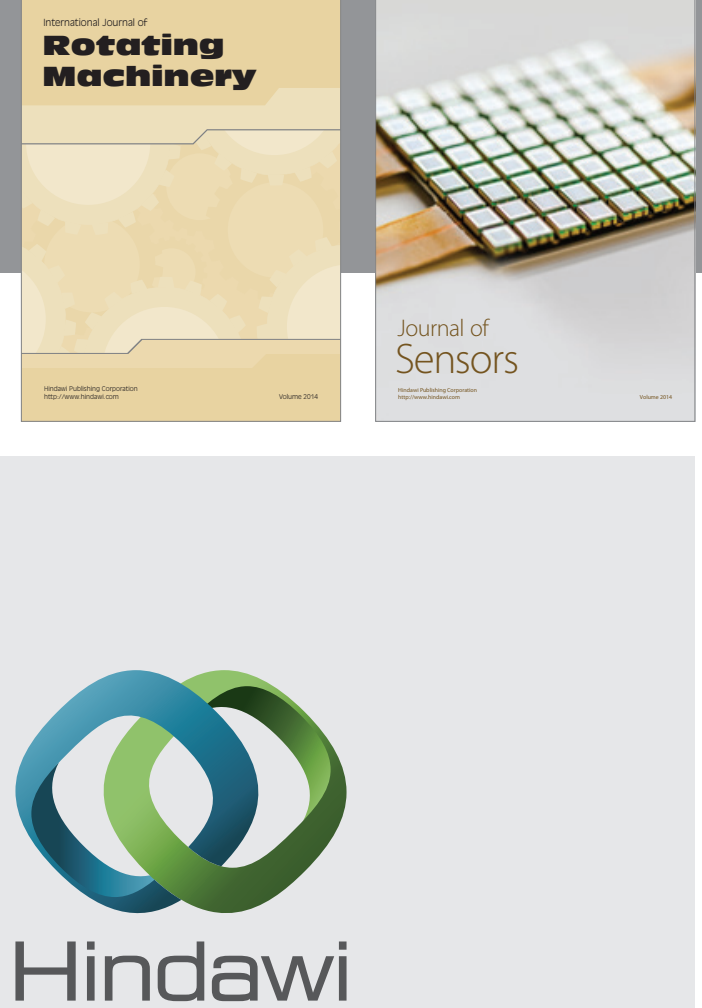

Submit your manuscripts at http://www.hindawi.com
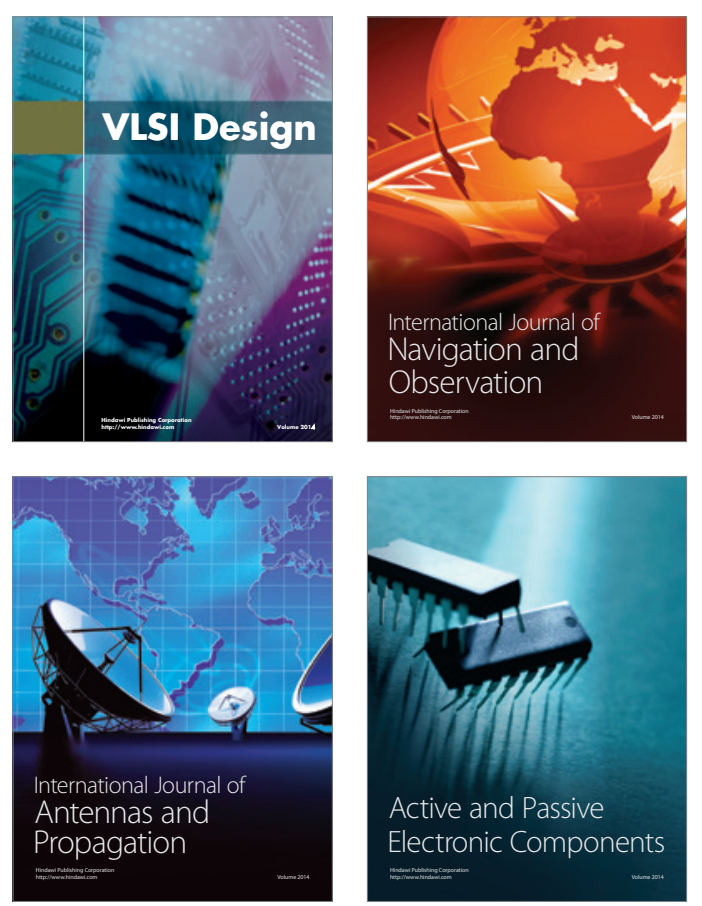
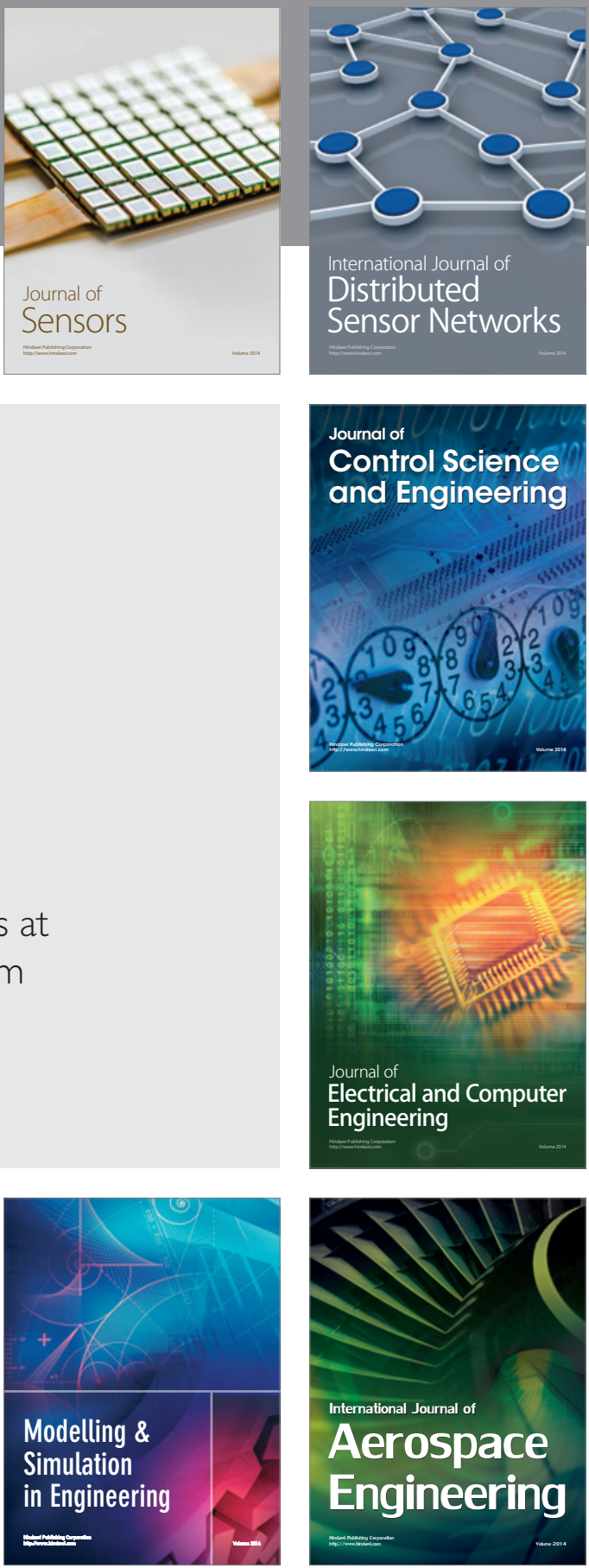

Journal of

Control Science

and Engineering
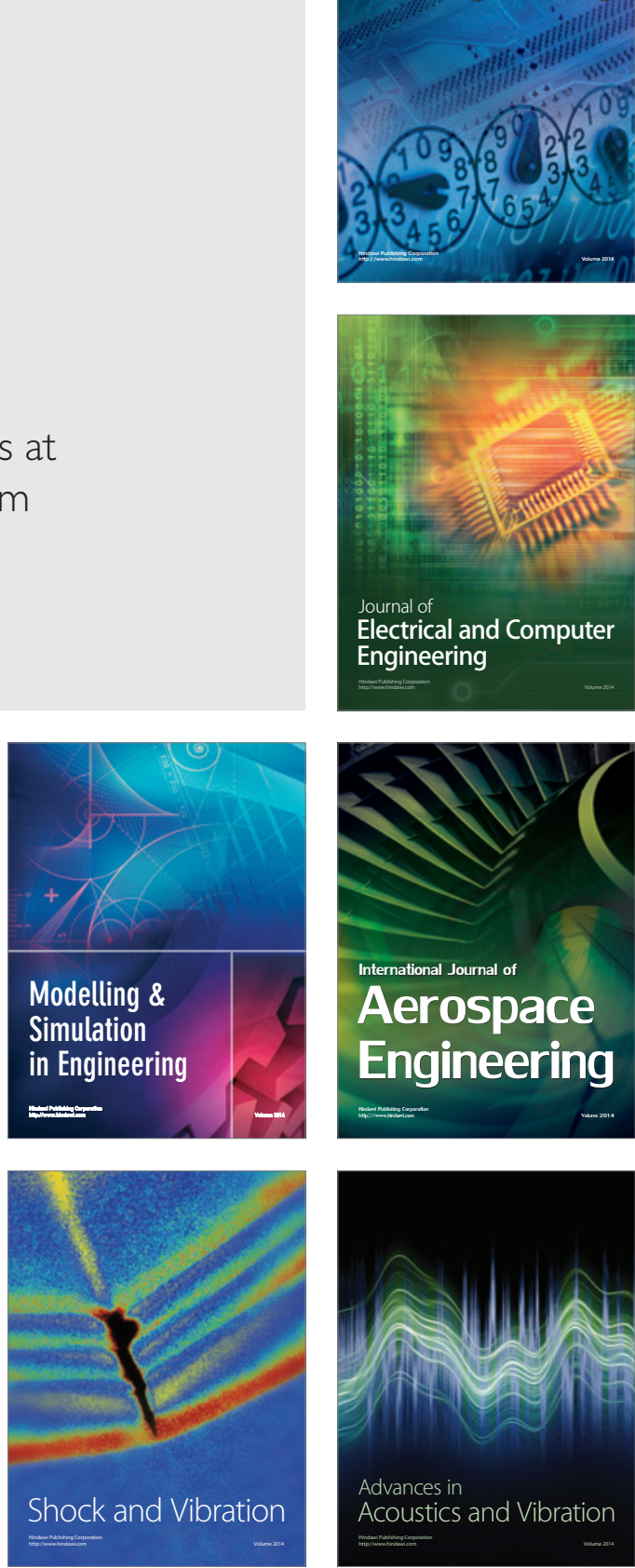